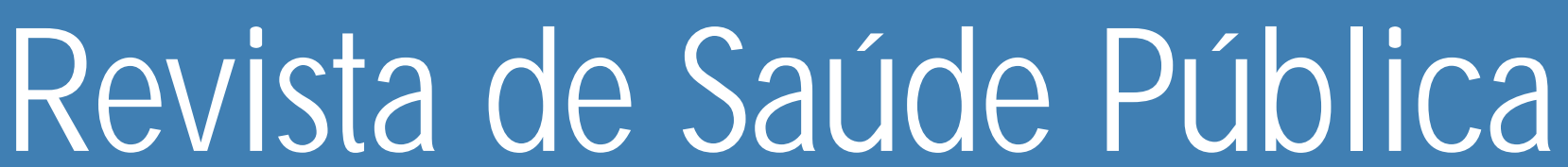

\begin{tabular}{lllllll}
\hline & 0 & $U$ & $R$ & $N$ & $A$ & $L$
\end{tabular}

$0 F$

P U

B L I C

H E A L T H

\title{
Atenção à saúde mental na rede básica: estudo sobre a eficácia do modelo assistencial
}

\section{Mental health care in health centers: study of the efficacy of the care given}

\section{Márcia A. do Amaral}

Departamento de Medicina Preventiva e Social da Faculdade de Medicina da U niversidade Estadual de Campinas. Campinas, SP - Brasil 


\title{
Atenção à saúde mental na rede básica: estudo sobre a eficácia do modelo assistencial
}

\author{
Mental health care in health centers: study of the \\ efficacy of the care given
}

Márcia A. do Amaral

Departamento de Medicina Preventiva e Social da Faculdade de Medicina da U niversidade Estadual de Campinas. Campinas, SP - Brasil

\begin{abstract}
Introdução

\section{Resumo}

Objetivou-se analisar a implementação da política de saúde mental para a rede básica de saúde, no Município de Campinas, SP (Brasil).
\end{abstract}

Material e Método

Resultados

Conclusões

Introduction

Material and Method

Foi feito estudo epidemiológico descritivo de uma amostra de 150 pacientes egressos de um hospital psiquiátrico e encaminhados aos centros de saúde para continuidade do tratamento. Durante 4 meses após a alta, foi verificado o comparecimento dos pacientes às atividades, assim como a ocorrência de reinternações.

Foram encontrados $48,6 \%$ dos pacientes em alta hospitalar que não demandaram atendimento nos centros de saúde e dos que o fizeram, 51,4\% abandonaram o tratamento num período de 4 meses. A prevalência de reinternações em 4 meses foi de $24,7 \%$, sendo maior entre os pacientes com diagnóstico de psicoses.

Foram evidenciados problemas na implementação da política de saúde mental para a rede básica relativos à definição das políticas, à organização do processo de trabalho das equipes de saúde e aos resultados alcançados. Foi referendado o diagnóstico de que a transformação do modelo manicomial demanda a existência de novos equipamentos de reabilitação psicossocial e a articulação intersetorial para alcançar seus objetivos de desospitalização e resgate da cidadania dos doentes mentais.

Avaliação de processos e resultados (cuidados de saúde). Serviços de saúde mental, organização e administração. Modelo assistencial.

\section{Abstract}

The implementation of the mental health policy in the health centers of Campinas, Southeastern Brazil, is analysed.

The methodology used consisted of a descriptive epidemiological study of a sample of 150 patients, discharged from a psychiatric hospital, and referred to health centers for continuing treatment. During the 4 months following discharge the attendance of the patients at these centers and return visits into hospital were verified.

\footnotetext{
* Resumo da dissertação de mestrado em Saúde Coletiva, apresentada à Faculdade de Ciências Médicas da UNICAMP, em 1995. Correspondência para/Correspondence to: Márcia A. do Amaral - Rua João Chati, 112 Casa 31 - Pq. Taquaral - 13087-200 Campinas, SP - Brasil. E-mail: marcia@mpc.com.br Edição subvencionada pela FAPESP. Processo 96/5999-9.

Recebido em 13.6.1996. Reapresentado em 31. 10.1996. Aprovado em 4.12.1996.
} 
Results The results showed that $48.6 \%$ of the patients discharged by hospitals did not turn up at the health centers. Of those who did get those, $51.4 \%$ abandoned their treatment before the end of the 4 months.The percentaje of $24.7 \%$ of the patients returned to hospital, most of them receiving a diagnosis of psychosis.

Conclusions The problems encountered in the implementation of the mental health policy in the health center networt in Campinas as regards the definition of the policy, the organization of the work of the professional teams and the results these services achieve, were brought out. The evidence that the transformation of the asylum calls for new psycho-social rehabilitation services and intersectional articulation to obtain good results in de-hospitalization and the salvaging of the citizenship of mentally-ill patients is reinforced.

Outcome and process assessment (health care). Mental health services, organization. Assistential model.

\section{INTRO DUÇÃO}

O presente trabalho foi desenvolvido a partir da necessidade de avaliação dos resultados da assistência prestada pelo Serviço de Saúde Dr. Cândido Ferreira (Campinas- SP) e da rede ambulatorial municipal, mais especificamente em relação à continuidade do tratamento após as internações psiquiátricas ali efetivadas, pois não existiam mecanismos de análise do impacto obtido pelo sistema de saúde mental, implantado a partir de 1989.

A redução da média de permanência hospitalar e do número de internações para pacientes com quadro agudo constituíam as prioridades da política de saúde mental para o Município de Campinas, SP, como conseqüência do seu engajamento nas diretrizes da Reforma Psiquiátrica Brasileira - desinstitucionalização, desospitalização e garantia dos direitos dos doentes mentais - enunciadas a partir da I Conferência Nacional de Saúde Mental, realizada em 1987, na Conferência de Caracas (OMS/OPS), em 1990, e referendadas na II Conferência Nacional de Saúde Mental, em 1992. No período de 1991 a 1993 foram desativados por atendimento de má qualidade, 510 leitos psiquiátricos na região de Campinas, sendo 474 no município, demonstrando a disposição dos gestores locais em transformar o modelo assistencial vigente.

A finalidade de reduzir-se o tempo de internação era basicamente a de afastar o paciente de sua rede social, por um curto período, e dar continuidade ao tratamento em nível ambulatorial. Todos os esforços para o alcance destes objetivos estavam sendo efetivados pela equipe da unidade de internação do Serviço de Saúde Dr. Cândido Ferreira, mas permane- cia a inquietação acerca da continuidade do tratamento nos outros níveis do sistema, sobretudo na rede básica, uma vez que essa recebia a maior parte dos pacientes pós-internação e não existia uma sistemática de avaliação no município.

Desenhado em princípio como um estudo epidemiológico descritivo, o projeto foi alterado a partir da obtenção dos resultados preliminares que sugeriam a alta complexidade existente em relação à implementação de novas diretrizes na busca de uma transformação efetiva do modelo assistencial predominante em saúde mental no Brasil. Foi então desenvolvida uma pesquisa qualitativa sobre as representações dos profissionais de saúde mental acerca das políticas propostas e sua adequação às necessidades identificadas por eles, além de características da organização do processo de trabalho das equipes assistenciais, a fim de analisar suas relações com a situação de tratamento pós-alta dos pacientes.

O modelo assistencial - no sentido usado por Campos $^{3}$ (1994)... "deve ser a relação cambiante e tensa entre o político e o técnico e que contemple a partir das necessidades sociais, diretrizes políticas e referenciais teóricos, além dos modos de gestão" preconizava a prestação de serviços de saúde mental por meio de equipes de pequeno porte, compostas por psiquiatra, psicólogo e assistente social, alocadas em $23(50 \%)$ centros de saúde municipais para atendimento de egressos dos hospitais psiquiátricos e da demanda espontânea ( apenas 4 unidades contavam também com terapeuta ocupacional); o atendimento às urgências e internações de até $72 \mathrm{~h}$ nos 32 leitos do Hospital da Pontifícia Universidade Católica de Campinas (PUCAMP); internações de pacientes em 
crise nos 14 leitos do Hospital de Clínicas da Universidade Estadual de Campinas (UNICAMP), no Serviço de Saúde Dr. Cândido Ferreira e demais hospitais privados da região.

A proposta da organização da assistência ambulatorial, a partir dos centros de saúde tinha como pressuposto a inserção da saúde mental na área mais ampla da saúde geral (Secretaria Municipal de Saúde de Campinas ${ }^{10}$, 1991), visando a dar-lhe um caráter de serviço preventivo e de atenção integral, mas atribuindo-lhe ao mesmo tempo a missão de atender os pacientes graves, egressos de hospitais psiquiátricos, evitar a internação e contribuir para a reabilitação psicossocial dos mesmos. Na verdade, o que ocorreu foi uma dissociação entre os propósitos e os meios, ou seja, a competência das equipes da rede básica para assistir a esta clientela do estudo não existia, ficando as diretrizes bastante gerais e sem um detalhamento para sua operacionalização, como mostrou a análise da pesquisa qualitativa.

O Serviço de Saúde Dr. Cândido Ferreira é um complexo de atenção à saúde mental, privado filantrópico, conveniado sob a forma de co-gestão com a Secretaria Municipal de Saúde de Campinas, desde 1990, constituindo-se referência para o sistema de saúde de Campinas e região. Possui 4 unidades assistenciais: Unidade de Internação de pacientes em crise, Hospital-dia, Núcleo de Oficinas de Trabalho e Unidade de Reabilitação de Moradores.

A clientela atendida é preferencialmente aquela com risco de internação psiquiátrica - psicóticos, alcoolistas, drogaditos e neuróticos graves - para as unidades ambulatoriais e de internação na crise. A população de moradores do serviço tem uma variedade maior de diagnósticos, uma vez que a maioria está internada há mais de 10 anos, como produto da política hegemônica de exclusão dos doentes mentais e dos deficientes físicos e mentais.

Cada uma destas unidades possui objetivos e resultados esperados bem definidos, tomando como referencial as necessidades de cada usuário e estruturando seu processo de trabalho para dar concretude às diretrizes institucionais: evitar o afastamento do doente do seu meio social, contribuir para ampliar esta rede de relações sociais, profissionalização, oferta de trabalho protegido, estabelecimento de vínculo com o serviço, respeito aos direitos de cidadania. $\mathrm{O}$ modelo de gestão pressupõe o trabalho em equipe com grande autonomia na sua organização, a interdisciplinaridade e a participação dos profissionais na definição das diretrizes. Existe uma circulação de pacientes entre as diferentes unidades, segundo a mudança nas suas necessidades e seus ganhos de autonomia para a vida diária. Algumas vezes o paciente com alta da unidade de internação é encaminhado para o Hospital-dia ou para o Núcleo de Oficinas de Trabalho. No entanto, a maioria dos pacientes é referenciada para a rede municipal de Centros de Saúde ou para um dos dois Centros de Apoio Psicossocial instalados a partir de 1993.

Um dado da realidade a ser considerado é que essas equipes possuiam, à época do estudo, pequena inserção na comunidade local e não contavam com o apoio de outros equipamentos de saúde mental na sua área de abrangência (oficinas terapêuticas e centros de convivência social).

As referências teóricas que norteiam o trabalho ali realizado têm suas raízes na experiência italiana, iniciada na década de 60 com a Comunidade Terapêutica, instalada em Gorizia, Itália*. O movimento denominado Psiquiatria Democrática partiu da constatação de que a psiquiatria serviu de instrumento de gestão da desordem e misérias sociais, tendo o hospital psiquiátrico como instrumento fundamental. A luta social (e interna à psiquiatria) desencadeada em várias regiões da Itália, culminou com a elaboração da Reforma Psiquiátrica (1978), com a proibição de internações em hospitais psiquiátricos (realizadas a partir de então em hospitais gerais) e a implementação de uma rede de serviços extra-hospitalares de saúde e ação social, incluindo-se moradias protegidas.

Os psiquiatras renovadores italianos criticavam o paradigma da psiquiatria como um conjunto de normas teóricas e ações para solucionar o problema da doença mental por meio da racionalidade positivista de combater o problema (doença mental) com uma solução (cura), sendo que para Rotelli ${ }^{9}$ (1990), a doença mental é bastante "não conhecível" e freqüentemente incurável, apesar dos esforços para darlhe uma explicação e definição racionais.

Abandonou-se então o paradigma que buscava a cura da doença mental para se buscar a "reinvenção da saúde" e a "reprodução social do paciente". Assim, não se valoriza de maneira absoluta o diagnóstico psiquiátrico e as causas das perdas pelas quais estaria passando o paciente em relação às suas funções psíquicas.Valoriza-se como objeto de intervenção a "existência-sofrimento dos pacientes" e suas formas de expressão dentro de cada contexto social (Rotelli ${ }^{9}$ 1990).

\footnotetext{
* Experiência iniciada por Franco Basaglia. Para maiores informações, ver “A Instituição Negada”, editada em 1985 pela Ed. Graal, Rio de Janeiro.
} 


\section{MATERIAL E MÉTO DO}

Para avaliar a continuidade do tratamento na rede ambulatorial foi selecionado um grupo de 150 pacientes com alta do Serviço de Saúde Dr. Cândido Ferreira, nos meses de junho, julho e agosto de 1992 e abril, maio e junho de 1993 e encaminhados aos centros de saúde da sua região de moradia. Esses pacientes constituíam a totalidade das altas de moradores de Campinas que foram referenciados para a rede básica de saúde.

Durante 4 meses após a alta foram registrados os comparecimentos aos serviços de referência, as reinternações ocorridas no período, a existência de agendamento de consulta previamente à alta hospitalar, assim como o tempo entre a alta e o agendamento do primeiro atendimento. Estas variáveis foram analisadas também considerando-se o diagnóstico psiquiátrico, segundo a Classificação Internacional das Doenças, $9^{\text {a }}$ Revisão. Foram selecionadas todas as unidades básicas com equipe de saúde e mental, excluindo-se as que estavam sem psiquiatras no período em estudo, num total de 17 unidades em 1992 e 21 em 1993.

Os dados foram coletados dos prontuários por meio de um instrumento específico para esta finalidade. Não houve consolidação dos dados por centro de saúde, uma vez que alguns deles receberam um pequeno número de encaminhamentos, o que poderia tornar a análise menos consistente.

Como foi referido na introdução, o presente estudo foi complementado por uma pesquisa qualitativa sobre as representações dos profissionais de saúde mental e a organização do processo de trabalho das equipes, servindo para subsidiar as conclusões finais e mantendo assim o caráter de síntese deste artigo.

\section{RESULTADOS}

\section{Características Demográficas da Amostra}

As características demográficas da amostra estudada podem ser verificadas nas Tabelas 1 e 2 .

Tabela 1 - Distribuição dos pacientes segundo 0 grupo-diagnóstico.

Table 1 - Distribution of patients by with diagnosis.

\begin{tabular}{lcc}
\hline Diagnóstico & No & $\%$ \\
\hline Psicoses & 80 & 53,3 \\
Alcoolismo & 30 & 20,0 \\
Neuroses & 22 & 14,7 \\
Dependência de drogas & 2 & 1,3 \\
Deficiência mental & 13 & 8,7 \\
Outros & 3 & 2,0 \\
\hline Total & 150 & 100,0 \\
\hline
\end{tabular}

\section{Número de Meses em Seguimento}

Na Tabela 3 verifica-se que 48,6\% dos pacientes não compareceram aos serviços de saúde no período de 4 meses após a alta, sendo a média de meses em seguimento de 1,38 para o total da amostra.
Tabela 2 - Distribuição dos pacientes segundo a idade e o sexo.

Table 2 - Distribution of patients by with age and sex.

\begin{tabular}{lrrrr}
\hline Idade & \multicolumn{2}{c}{ Sexo } & \multicolumn{2}{c}{ Total } \\
\cline { 2 - 4 } & \multicolumn{1}{c}{$\mathrm{M}$} & \multicolumn{1}{c}{$\mathrm{F}$} & \multicolumn{2}{c}{} \\
\cline { 2 - 5 } & $\mathrm{N} 0$ & $\mathrm{~N} 0$ & $\mathrm{~N}$ o & $\%$ \\
\hline $1<20$ & 2 & 5 & 7 & 4,7 \\
$21-30$ & 16 & 16 & 32 & 21,6 \\
$31-40$ & 25 & 22 & 47 & 31,8 \\
$41-50$ & 26 & 14 & 40 & 27,0 \\
$>/ 51$ & 12 & 10 & 22 & 14,9 \\
\hline Total & 81 & 67 & 148 & 100,0 \\
\hline
\end{tabular}

Quando comparados os grupos de pacientes com alta em 1992 com os saídos em 1993, verifica-se que não há melhora na adesão ao tratamento. A média de meses em seguimento, em 1992, foi de 1,30 e, em 1993, de 1,55, não havendo diferença estatisticamente significante.

Tabela 3 - Pacientes que demandaram consultas na rede básica (pelo menos 1 consulta no período de 4 meses após a alta).

Table 3 - Patients who required consultation in health centers (at least 1 consultation withen 4 months after discharge).

\begin{tabular}{|c|c|c|c|c|c|c|}
\hline \multirow{2}{*}{$\begin{array}{l}\text { Consulta na } \\
\text { rede básica }\end{array}$} & \multicolumn{2}{|c|}{1992} & \multicolumn{2}{|c|}{1993} & \multicolumn{2}{|c|}{ Total } \\
\hline & $\bar{N} \underline{0}$ & $-\overline{\%}$ & $\bar{N} 0$ & \% & $\bar{N} \underline{0}$ & $-\overline{\%}$ \\
\hline Sim & 48 & 52,7 & 24 & 49,0 & 72 & 51,4 \\
\hline Não & 43 & 47,3 & 25 & 51,0 & 68 & 48,6 \\
\hline Total & 91 & 100,0 & 49 & 100,0 & 140 & 100,0 \\
\hline
\end{tabular}

$X 2=2,223 p>0,05$

Foram excluídos desta análise 10 pacientes por insuficiência dos dados.

$\mathrm{Na}$ Tabela 4 verifica-se que entre aqueles pacientes que iniciaram o seguimento na unidade básica de saúde, $51,40 \%$ abandonaram o tratamento num período de 4 meses após a alta, sendo que não houve diferença estatisticamente significante entre os anos em estudo.

Tabela 4 - Pacientes que abandonaram o tratamento no período de 4 meses de seguimento após a alta hospitalar (entre os que fizeram ao menos 1 consulta).

Table 4 - Patients who abandoned their treatment in the 4 months after discharge (of those had at least 1 consultation).

\begin{tabular}{|c|c|c|c|c|c|c|}
\hline \multirow{2}{*}{ Abandono } & \multicolumn{2}{|c|}{1992} & \multicolumn{2}{|c|}{1993} & \multicolumn{2}{|c|}{ Total } \\
\hline & $\bar{N} 0$ & $-\overline{\%}$ & $\bar{N} \underline{0}$ & \% & $\overline{\mathrm{N}} \underline{0}$ & $\overline{\%}$ \\
\hline Sim & 27 & 56,3 & 10 & 41,7 & 37 & 51,4 \\
\hline Não & 21 & 43,7 & 14 & 58,3 & 35 & 48,6 \\
\hline Total & 48 & 100,0 & 24 & 100,0 & 72 & 100,0 \\
\hline
\end{tabular}

Na Tabela 5 encontraram-se as médias e os desvios-padrão do número de meses de seguimento, entre aqueles pacientes que iniciaram o tratamento na unidade básica de saúde. Os dados mostram que existe diferença estatisticamente significante entre os 
anos em estudo, tendo aumentado a média de meses em seguimento para os pacientes com alta em 1993. Apesar deste resultado, não ocorreu mudança no perfil de adesão geral ao serviço de saúde mental.

Tabela 5 - M édias e desvios-padrão do número de meses de seguimento, segundo 0 ano de alta.

Table 5 - Averages and standard deviations of the number of months of follow-up by year of discharge.

\begin{tabular}{ccc}
\hline Ano & Média & Desvio-padrão \\
\hline 1992 & 2,46 & 1,44 \\
1993 & 3,17 & 1,09 \\
\hline $\mathrm{t}=2,12 \mathrm{p}=0,03$ & &
\end{tabular}

\section{Consultas Agendadas Previamente à Alta Hospitalar}

Estava normatizado pela Secretaria de Estado da Saúde de São Paulo, o agendamento no serviço ambulatorial previamente à alta hospitalar, com o intuito de contribuir com a continuidade do tratamento.

Verificou-se que $67,3 \%$ dos pacientes receberam alta com agendamento da consulta na unidade de referência para seu local de moradia. Esta variável não se diferenciou estatisticamente entre os anos de 1992 e 1993.

Relacionando-se a realização de ao menos uma consulta no centro de saúde à existência ou não de agendamento prévio,verificou-se que $54,2 \%$ dos pacientes sem agendamento não compareceram ao serviço e para aqueles com agendamento a cifra foi de $46,0 \%$, não havendo diferença estatisticamente significante entre os dois grupos.

\section{Tempo D ecorrido Entre a Alta H ospitalar e a Consulta Ambulatorial}

O tempo médio decorrido entre a alta hospitalar e a consulta ambulatorial esteve em 15,0 dias, tendo baixado de $1992(16,8)$ para $1993(11,2)$, sendo a diferença estatisticamente significante. Os tempos variaram entre um e 48 dias. A mediana para 1992 foi de 13 dias, para 1993 de 9 e para o total da amostra de 12. Não se encontrou diferença estatística no número de meses em seguimento entre os grupos que tiveram suas consultas agendadas para mais ou me$\operatorname{nos} 7 \operatorname{dias}(\mathrm{X} 2=3,22 \mathrm{p}>0,05)$.

\section{Reinternações}

Ocorreram 37 reinternações num período de até 4 meses após a alta em estudo, representando $24,7 \%$ do total de pacientes da amostra. Não houve diferença estatisticamente significante entre os grupos com alta em 1992 e 1993, cujos percentuais de reinternação foram de 25,2 e 23,5, respectivamente.
Relacionando-se a reinternação com o acompanhamento no centro de saúde, verificou-se que $28,0 \%$ dos que não compareceram foram reinternados contra $14,3 \%$ dos que compareceram durante 4 meses após a alta, sem associação estatisticamente significante entre as variáveis $(\mathrm{X} 2=2,397 \mathrm{p}>0,05)$.

O tempo médio entre a alta hospitalar em estudo e as reinternações foi de 34,2 dias, sendo que para o grupo com alta em 1992 foi de 29,2 dias e a mediana foi de 18 dias. Para o grupo de 1993, o tempo médio foi de 43,5 dias e a mediana de 38 , demonstrando aumento do período ocorrido entre as internações, sendo a diferença das médias estatisticamente significante $(p=0,043)$.

\section{Prevalência de Reinternações por G rupos Diagnósticos}

A maior prevalência de reinternações no período de 4 meses após a alta ocorreu entre os pacientes psicóticos (32,5\%), sendo que os alcoolistas ou com outros diagnósticos foram reinternados em menor percentagem $(15,7 \%)$, como mostra a Tabela 6.

Tabela 6 - O corrência de reinternações segundo o diagnóstico.

Table 6 - Incidence of new internment by diagnosis.

\begin{tabular}{|c|c|c|c|c|c|c|}
\hline \multirow{3}{*}{ Reinternações } & \multicolumn{4}{|c|}{ Diagnóstico } & \multirow{2}{*}{\multicolumn{2}{|c|}{ Total }} \\
\hline & \multicolumn{2}{|c|}{ Psicoses } & \multicolumn{2}{|c|}{ O utros } & & \\
\hline & № & $\%$ & No & $\%$ & No & $\%$ \\
\hline Sim & 26 & 32,5 & 11 & 15,7 & 37 & 24,7 \\
\hline Não & 54 & 67,5 & 59 & 84,3 & 113 & 75,3 \\
\hline Total & 80 & 100,0 & 70 & 100,0 & 150 & 100,0 \\
\hline
\end{tabular}

\section{Número de Meses em Seguimento na U nidade Ambulatorial Segundo o G rupo-D iagnóstico}

Os pacientes não psicóticos têm aparentemente menor grau de adesão aos serviços ambulatoriais (52,9\% de nenhum comparecimento) do que os pacientes com quadros psicóticos $(44,9 \%)$. Contudo, não foi encontrada associação estatística entre o número de meses em seguimento ambulatorial e o diagnóstico, como mostra a Tabela 7.

Tabela 7- Número de meses em seguimento, segundo o diagnóstico.

Table 7 - N umber of months follow-up by diagnosis.

\begin{tabular}{|c|c|c|c|c|c|c|}
\hline \multirow{2}{*}{$\begin{array}{l}\mathrm{N} \text { o de meses } \\
\text { em seguimento }\end{array}$} & \multicolumn{2}{|c|}{ Psicoses } & \multicolumn{2}{|c|}{$\begin{array}{l}\text { Demais } \\
\text { patologias }\end{array}$} & \multicolumn{2}{|c|}{ Total } \\
\hline & $\mathrm{N} 0$ & $\%$ & $\mathrm{~N} 0$ & $\%$ & № & $\%$ \\
\hline 0 & 35 & 44,9 & 37 & 52,9 & 72 & 48,6 \\
\hline $1-3$ & 19 & 24,3 & 22 & 31,4 & 41 & 27,7 \\
\hline 4 & 24 & 30,8 & 11 & 15,7 & 35 & 23,7 \\
\hline Total & 78 & 100,0 & 70 & 100,0 & 148 & 100,0 \\
\hline
\end{tabular}




\section{DISCU SSÃO}

Pensar a desospitalização e a reabilitação psicossocial do doente mental implica o estabelecimento de modelos assistenciais e práticas de saúde coerentes com as diretrizes políticas (Testa $\left.{ }^{11}, 1992\right)$. Os modelos assistenciais em saúde têm que ser estruturados de modo a viabilizarem a missão institucional e atenderem às necessidades da clientela. (Campos ${ }^{3}$, 1994).

Em Campinas, a atribuição de competência à rede básica e equipes de saúde mental, de pequeno porte, de assistir pacientes egressos de hospital psiquiátrico, revelou-se ineficaz para o alcance dos objetivos de desospitalizar e desinstitucionalizar nos seguintes aspectos, considerados pressupostos de qualidade por Donabedian apud Nogueira ${ }^{7}, 1994$ : aceitabilidade, eqüidade e eficácia.

Quanto à Aceitabilidade, ou seja, a conformidade com as preferências do paciente no que concerne à acessibilidade, relação médico-paciente e melhoras obtidas, o presente estudo mostrou que a clientela definida como preferencial não reconhecia a rede básica como alternativa para a resolução de seus problemas de saúde, uma vez que 48,6\% dos pacientes egressos do Serviço de Saúde Dr. Cândido Ferreira não demandaram atendimento ambulatorial após a alta. Mesmo os pacientes vinculados aos serviços ambulatoriais encontraram dificuldades para a manutenção do tratamento relacionadas ao acesso e resolutividade dos mesmos, traduzidas na taxa de abandono de $51,4 \%$, no período de 4 meses após a alta.

A aparente contradição entre a demanda atendida pelos serviços e as prioridades estabelecidas pela Secretaria Municipal de Saúde, Campinas, torna necessária a reflexão sobre o processo de produção de demandas, inclusive pelos serviços de saúde mental. Quem demanda um determinado serviço já foi convencido pelo mesmo que ali encontrará a resposta para a sua necessidade (Baremblit ${ }^{2}$, 1994).

Através da práticas e das condições de trabalho oferecidas os serviços acabam conformando uma determinada demanda que, posteriormente, pode ser declarada como equivocada ou fora de lugar. $\mathrm{O}$ fato de que vários psicólogos atendiam exclusivamente crianças, ou de que os médicos realizavam apenas consultas para prescrição de medicação, ou dos alcoolistas e psicóticos não terem comparecido ao serviço para o tratamento nos mostra quais eram os grupos que acreditavam que seus problemas seriam resolvidos nos centros de saúde.

Em relação à Eficácia (capacidade do cuidado, assumindo sua forma mais perfeita, de contribuir para a melhoria das condições de saúde) para evitar a internação psiquiátrica, os dados mostraram que $32,5 \%$ dos pacientes psicóticos foram reinternados num período de até 4 meses após a alta. No ano de 1993 ocorreu aumento do tempo decorrido entre a alta em estudo e a reinternação, mas a prevalência da reinternação não se alterou. Ainda que não existissem à época do estudo, metas ou parâmetros para as reinternações, este dado pode ser considerado desfavorável enquanto resultado, devendo ser monitorado para aprofundamento da análise.

Em relação à Eqüidade (igualdade na distribuição do cuidado e de seus efeitos sobre a saúde) enquanto resultado esperado, pode-se considerar não atingido, porque para uma mesma clientela, definida como prioritária, os recursos terapêuticos oferecidos foram diversos do ponto de vista da tecnologia empregada e da resolutividade alcançada. Ou seja, dependendo das características da unidade de saúde onde o tratamento era prestado, o paciente recebia atenção em saúde mental de diferentes qualidades. As modalidades de atenção podiam variar desde apenas consulta médica para atualização da medicação, até atendimento multiprofissional e participação em oficinas profissionalizantes.

A pesquisa qualitativa realizada com profissionais de 5 unidades de saúde, totalizando 14 entrevistas, mostrou que além de problemas com os resultados da política, outras dificuldades existiram.

A primeira diz respeito à definição da políticas de saúde mental.

A definição da prioridade de atendimento nos centros de saúde aos pacientes mais graves e egressos de hospitais não contemplou a necessidade de atendimento da demanda por problemas de menor gravidade e merecedora de uma resposta institucional. Esta pressão gerada nos serviços contribuiu para que os profissionais de saúde tivessem dificuldade de lidar com a demanda "oficial" e a real, influindo na organização do processo de trabalho. Dados do estudo multicêntrico de morbidade psiquiátrica em áreas urbanas brasileiras, realizado por Almeida Filho e col. ${ }^{1}(1992)$ mostram a diversidade dos perfis de morbidade, dependendo das diferenças socioculturais e níveis de desenvolvimento das cidades. Analisando-se os dados, por grupo-diagnóstico, os autores encontraram que a ansiedade e as fobias constituem os principais problemas de saúde mental da população urbana brasileira. Para agravar esta situação, ocorreu a pouca definição das diretrizes operacionais, ou o como fazer. Atendimentos individuais ou em grupos, características da clientela dos grupos, psiquiatria clínica, atendimento exclusivo a crianças ou a adultos, modos de agendamento e ou- 
tros são questões que remetem a tecnologias diferenciadas e com experiências concretas existentes que poderiam subsidiar as escolhas. Para Gonçalves ${ }^{5}$ (1992) "conceitualmente, 'modelo' aponta para a necessidade ineludível de consistência prática entre objetos do trabalho, instrumentos e a ação do agente do trabalho, para que o processo possa efetivamente objetivar-se num produto". Os dados da pesquisa mostraram a relação existente entre a clareza da política e a incorporação das diretrizes no processo de trabalho das equipes.

O último ponto das políticas e talvez o mais importante, é a estratégia adotada para a organização da rede de serviços de saúde mental, ou seja, a opção pela instalação das equipes em centros de saúde. Apesar de não ter sido considerada problema pelo conjunto dos profissionais entrevistados, a racionalização de recursos disponíveis, mediante à concentração dos mesmos em um número menor de unidades, poderia aumentar a resolutividade do modelo (desde que fossem equacionados problemas de acesso dos pacientes e da ausência de oficinas ou centros de convivência) .

Outra questão foi a ausência de propostas de articulação intersetorial, que diminuiu a viabilidade de instalação de equipamentos de reabilitação, como as oficinas, centros de convivência e moradias protegidas.

A segunda dificuldade encontrada foi relativa à organização do processo de trabalho das equipes.

A ausência de uma sistemática de planejamento para o nível local fez com que o trabalho fosse estruturado sobre o atendimento da demanda espontânea e, em menor escala, da demanda de egressos dos hospitais. Uma das expressões deste problema foi a demora no agendamento da consulta pós-alta com média de 15 dias de espera para o total da amostra. O reconhecimento dessa demanda também foi apresentado como um problema que dificultou a estruturação do serviço para atender às necessidades dos pacientes. Uma das unidades estudadas estava em fase de implantação de um instrumento de coleta de dados sobre o perfil da clientela de sua área de cobertura para redirecionar as ações ali desenvolvidas, segundo as necessidades dos usuários. Da mesma forma, a ausência de supervisão que instrumentalizasse as equipes para o processo de gestão de suas práticas, favoreceu a atuação individualizada dos profissionais, sem que a instituição apresentasse proposta de petição e prestação de contas.

A definição dos papéis dentro das equipes, no geral manteve a divisão tradicional entre o trabalho do médico e dos não-médicos, com exceção de uma unidade, onde o trabalho se dava efetivamente em equipe. E, finalmente, o baixo envolvimento das equipes com o funcionamento global das unidades e a articulação entre as áreas clínicas prejudicaram a atenção integral aos problemas de saúde dos usuários. Considerando que um dos objetivos da instalação das equipes de saúde mental, nos centros de saúde, era a facilitação da atenção integral, os dados revelaram as dificuldades para a sua consecução.

Frente a todas essas questões, seria esperada uma baixa legitimidade social do modelo, até mesmo pelo pequeno envolvimento com outras organizações da sociedade.

A implementação de uma nova política pública, com as implicações socioculturais da proposta da reforma psiquiátrica brasileira passa pela necessidade de articulação intersetorial e por ações que contribuam para alterar as representações sociais sobre a loucura e os direitos dos doentes mentais.

A nova abordagem da loucura proposta pela psiquiatria democrática italiana poderia ter sido em Campinas um dispositivo, algo que tornasse possível uma mudança cultural de maneira mais efetiva.

A transformação do modelo assistencial de um conceito teórico em diretrizes operacionais é um processo complexo e depende de vários fatores para que se mantenha a direcionalidade pretendida pelos diferentes atores sociais participantes do jogo social (Matus ${ }^{6}, 1993$ ).

Esta mutação do político em técnico, ou de um dispositivo em equipamento de saúde não pode deixar de construir o sujeito histórico. A participação dos usuários nas explicitações de suas necessidades e na avaliação de sua satisfação praticamente não existia, assim como os profissionais de saúde mental estavam descontentes, mas uma parcela deles acomodados em suas práticas.

Apesar da histórica escassez de recursos destinados à saúde, foram evidenciadas várias situações problemáticas no campo gerencial que poderiam ser melhoradas com os recursos existentes. A tecnologia disponível na área de planejamento e avaliação de serviços de saúde tem mostrado bons resultados quando aliada à vontade política de mudar a situação.

Em conclusão, o modelo assistencial proposto inicialmente em Campinas apresenta várias dificuldades operacionais, com dispersão de recursos, demanda de gerenciamento bastante competente para articular vários serviços de baixa complexidade tecnológica e com a missão abrangente de atender demandas espontânea e referenciada.O presente trabalho pode contribuir para a reelaboração do papel dos diferentes equipamentos de saúde mental que compõem o sistema público. 


\section{REFERÊN CIAS BIBLIO G RÁFICAS}

1. ALMEIDA FILHO, N.; MARI, J.J.; COUTINHO, E.; FRANÇA, J.F.; FERNANDES, J.G.; ANDREOLI, S.B.; BURNELLO, E.D. Estudo multicêntrico de morbidade psiquiátrica em áreas urbanas brasileiras (Brasília, São Paulo, Poro Alegre). Rev. ABP-APAL, 14: 93-104, 1992.

2. BAREMBLIT, G. Compêndio de análise institucional e outras correntes: teoria e prática. Rio de Janeiro, Ed. Rosa dos Tempos, 1994.

3. CAMPOS, G.W.S. Sobre a reforma dos modelos de atenção: um modo mutante de fazer saúde. Campinas. Fac. de Ciências Médicas da UNICAMP, 1994.

4. CAMPOS, G.W.S. Considerações sobre a arte e a ciência da mudança: revolução das coisas e reformas das pessoas; o caso da saúde. In: Cecílio, L.C.O. Inventando a mudança na saúde. São Paulo, Ed. Hucitec, 1994.
5. GONÇALVES, R.B.M. Práticas de saúde: processos de trabalho e necessidades. São Paulo, Departamento de Medicina Preventiva da Fac. Med. USP, 1992 [no prelo].

6. MATUS, C. Política, planejamento e governo. Brasília, IPEA, 1993.

7. NOGUEIRA, R.P. Perspectivas da qualidade em saúde. Rio de Janeiro, Ed.Qualitymark, 1994.

8. ORGANIZACION PANAMERICANA DE LA SALUD. Reestruturación de la atención psiquiátrica: bases conceptuales y guias para su implementación. Washington, D.C.,1991.

9. ROTELLI, F. et al.Desinstitucionalização. São Paulo, Ed. Hucitec, 1990.

10. SECRETARIA MUNICIPAL DE SAÚDE DE CAMPINAS. Projeto do hospital-dia. Campinas, 1991.

11. TESTA, M. Pensar em saúde. Porto Alegre, Artes Médicas, 1992. 